\title{
Direct effects of ciliary neurotrophic factor on brown adipocytes: evidence for a role in peripheral regulation of energy homeostasis
}

\author{
V Ott, M Fasshauer ${ }^{1}$, A Dalski, H H Klein and J Klein \\ Department of Internal Medicine I, Medical University of Lübeck, Ratzeburger Allee 160, 23538 Lübeck, Germany \\ ${ }^{1}$ Department of Internal Medicine III, University of Leipzig, 04103 Leipzig, Germany \\ (Requests for offprints should be addressed to J Klein; Email: johannes.klein@medinf.mu-luebeck.de)
}

\begin{abstract}
Ciliary neurotrophic factor (CNTF) plays an important role in regulating neuronal growth. Recently, central anorexigenic effects of this cytokine have been characterized. However, peripheral effects on tissues that actively contribute to the regulation of energy homeostasis have not been described. Here, we report direct potent and selective effects of CNTF on growth factor and metabolic signalling intermediates in mouse brown adipocytes. CNTF stimulates STAT3, MAP kinase, Akt, and p70 S6 kinase. We find that, next to mediating Akt and p70 S6
\end{abstract}

kinase activation, both phosphatidylinositol 3-kinase and protein kinase $\mathrm{C}$ are separately acting, main intermediates for inducing mitogen-activated protein (MAP) kinase activation. On a functional level, CNTF enhances $\beta 3$ adrenergic induction of uncoupling protein-1. Thus, these results demonstrate direct effects of CNTF on adipose tissue signalling and metabolism and suggest a novel role for this cytokine in the peripheral regulation of energy homeostasis.

Journal of Endocrinology (2002) 173, R1-R8

\section{Introduction}

Ciliary neurotrophic factor (CNTF) is an important trophic factor for neuronal cell growth and differentiation (Sleeman et al. 2000). Studies in rodents and humans have shown that CNTF treatment results in decreased food intake and weight loss (Gloaguen et al. 1997, ALS CNTF Treatment Study Group 1996). They suggest an anorexigenic role of this factor in the central nervous system regulation of body weight (Gloaguen et al. 1997, Lambert et al. 2001) involving an inhibition of the orexigenic Neuropeptide $\mathrm{Y}$ in the hypothalamus (Xu et al. 1998).

It is unknown whether direct CNTF effects on peripheral tissues contribute to the regulation of body weight and energy homeostasis. We have previously shown that brown adipocytes provide an excellent model for the study of molecular mechanisms contributing to the control of energy balance (Klein et al. 1999, Klein et al. 2000). Here, we demonstrate that CNTF directly and selectively activates important growth factor and metabolic signalling pathways in brown adipocytes and enhances $\beta 3-$ adrenergic stimulation of uncoupling protein-1, thus providing evidence for a peripheral role of this anorexigenic peptide in the regulation of energy homeostasis.

\section{Materials and Methods}

\section{Materials}

Phosphospecific antibodies against the following molecules were employed for immunoblotting: STAT3 (Tyr 705), p42/p44 mitogen activated protein (MAP) kinase, Akt (Ser 473) and p70 S6 kinase (Thr421/Ser424) (all from Cell Signalling Technology, Inc., Beverly, MA, USA). Recombinant rat CNTF was purchased from PeproTech Inc., Rocky Hill, NJ, USA. The pharmacological inhibitors GF109203X, LY294002 and Ag490 were obtained from Calbiochem, La Jolla, CA, USA, PD98059 and Rapamycin from Cell Signalling Technology, Inc. (Beverly, MA, USA). Antibodies directed against uncoupling protein-1 (UCP-1) were from Alpha Diagnostic International (San Antonio, TX, USA). Unless stated otherwise, all other chemicals were purchased from Sigma-Aldrich Co. (St Louis, MO, USA).

\section{Cell culture and Western blotting}

SV40T-immortalized mouse brown adipocytes were cultured as previously described (Klein et al. 1999) and used 
between passages 10 and 25 . Fully differentiated cells were starved for a period of 24 to $48 \mathrm{~h}$ in serum-free medium prior to carrying out the experiments. Following treatment with inhibitors and CNTF as indicated, cells were lysed, and normalized protein lysates were immunoblotted with the respective antibodies and transferred to nitrocellulose membranes (Schleicher and Schuell Inc.; Keane, NH, USA) essentially as described (Klein et al. 1999). Bands were visualized using enhanced chemiluminescence (Roche Molecular Biochemicals, Mannheim, Germany).

\section{Analysis of UCP-1 gene expression}

We used quantitative real-time RT-PCR in a fluorescent temperature cycler (LightCycler, Roche Molecular Biochemicals, Mannheim, Germany) to assess UCP-1 gene expression, essentially as described previously (Fasshauer et al. 2001b, 2001c). Briefly, total RNA was isolated from brown adipocytes using RNAwiz reagent (Ambion, Austin, TX), and $5 \mu \mathrm{g}$ of total RNA was reverse transcribed using Superscript II RNase $\mathrm{H}^{-}$Reverse Transcriptase (Invitrogen Life Technologies, Karlsruhe, Germany). $10 \%$ of each RT reaction was amplified in a $20 \mu \mathrm{l}$ PCR containing $2.5 \mathrm{mM} \mathrm{MgCl} 2,250 \mathrm{nM}$ of each primer and 1X QuantiTect SYBR Green PCR-Mix (QIAGEN, Hilden, Germany). Samples were loaded into capillary tubes and incubated in the Lightcycler for an initial denaturation at $95^{\circ} \mathrm{C}$ for $900 \mathrm{~s}$, followed by 55 cycles, each cycle consisting of $95{ }^{\circ} \mathrm{C}$ for $10 \mathrm{~s}, 56{ }^{\circ} \mathrm{C}$ for $10 \mathrm{~s}$, and $72{ }^{\circ} \mathrm{C}$ for $15 \mathrm{~s}$. The following oligonucleotide primers were used: UCP-1 (accession no. M21222+ M21244) ATG GTG AAC CCG ACA ACT TCC GAA GTG (sense) and GTA CTG GAA GCC TGG CCT TCA CCT TGG (antisense), hypoxanthine guanine phosphoribosyl transferase (HPRT) (accession no. NM_013556) GTT GGA TAC AGG CCA GAC TTT GT (sense) and CAC AGG ACT AGA ACA CCT GC (anti-sense). Cycle-to-Cycle SYBR Green I fluorescence emission readings were monitored. Quantification was done by using the second derivative maximum method of the LightCycler Software (Roche Molecular Biochemicals, Mannheim, Germany) which determines the crossing points of individual samples by an algorithm identifying the first turning point of the fluorescence curve. This turning point corresponds to the first maximum of the second derivative curve and correlates inversely to the log of the initial template concentration. UCP-1 mRNA levels were normalized to those of HPRT. Amplification of specific transcripts was confirmed by producing melting curve profiles (cooling the sample to $65{ }^{\circ} \mathrm{C}$ for $10 \mathrm{~s}$ and heating slowly to $95^{\circ} \mathrm{C}$ in steps of $0 \cdot 2{ }^{\circ} \mathrm{C} / \mathrm{s}$ with continuous measurement of fluorescence) at the end of each run.

\section{Statistical analysis}

'Sigma Plot'- software (SPSS Science; Chicago, IL, USA) was employed for statistical analysis of all data. Results are presented as mean $+/-$ S.E. Unpaired student's $t$-test was used for determination of statistical significance. $P$ values $<0 \cdot 05$ are considered significant, those $<0 \cdot 01$ highly significant.

\section{Results}

CNTF stimulates STAT3 phosphorylation in brown adipocytes

Treatment of brown adipocytes with CNTF resulted in a dose- and time-dependent induction of STAT3 phosphorylation with a maximal seven-fold increase at a concentration of $10 \mathrm{nM}$ after $10 \mathrm{~min}$ (Fig. $1 \mathrm{~A}$ and $\mathrm{B}$ ).

\section{CNTF induces MAP kinase phosphorylation}

MAP kinase is an important signalling molecule mediating control of gene transcription in response to growth factors. CNTF treatment potently induced p42/p44 MAP kinase phosphorylation (Fig. 2A and B), but not p38 MAP kinase phosphorylation (data not shown). The effect on $\mathrm{p} 42 / \mathrm{p} 44$ MAP kinase was dose- and time-dependent with a maximal approximately four-fold stimulation at a CNTF concentration of $10 \mathrm{nM}$ (Fig. 2A and B).

\section{MAP kinase phosphorylation by CNTF is mediated via} several independent pathways involving PI3-kinase and PKC

Inhibition of Janus kinase 2 (JAK2), a key proximal signalling element for STAT signalling, by Ag490 decreased the CNTF-induced MAP kinase phosphorylation by $45 \%$ (Fig. 2C). As expected, pretreatment of brown adipocytes with PD98059, a selective MEK kinase inhibitor, completely abrogated the stimulatory CNTF effect (Fig. 2C). Interestingly, the selective phosphatidylinositol 3 (PI3) -kinase inhibitor LY294002 $(10 \mu \mathrm{M})$ diminished the CNTF effect on MAP kinase by $50 \%$ (Fig. 2D). Similarly, treatment of cells with the protein kinase C (PKC) inhibitor GF109203X $(5 \mu \mathrm{M})$ also reduced the CNTF effect by approximately 55\% (Fig. 2D). Moreover, concurrent inhibition of both PI3-kinase and PKC completely abrogated the positive CNTF effect on MAP kinase phosphorylation, thus suggesting an additive effect of both signalling molecules (Fig. 2D).

\section{CNTF stimulates Akt phosphorylation in a PI3-kinase- and PKC-dependent but $m$ Tor-independent manner}

Akt is a main signalling element most likely involved in mediating metabolic cellular responses such as glucose transport. CNTF stimulation resulted in a dose- and time-dependent increase in Akt phosphorylation, with a three- to five-fold stimulation at a CNTF-concentration of $10 \mathrm{nM}$ after $10 \mathrm{~min}$ (Fig. 3A and B). JAK2 inhibition with 
A

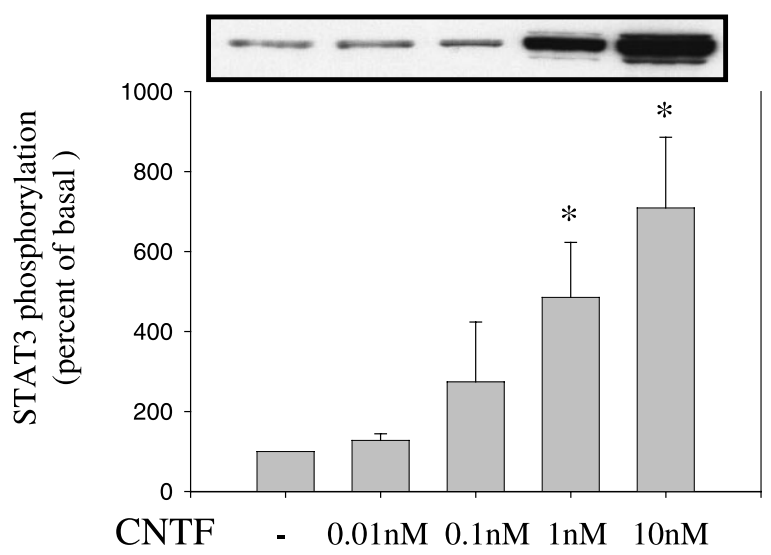

B

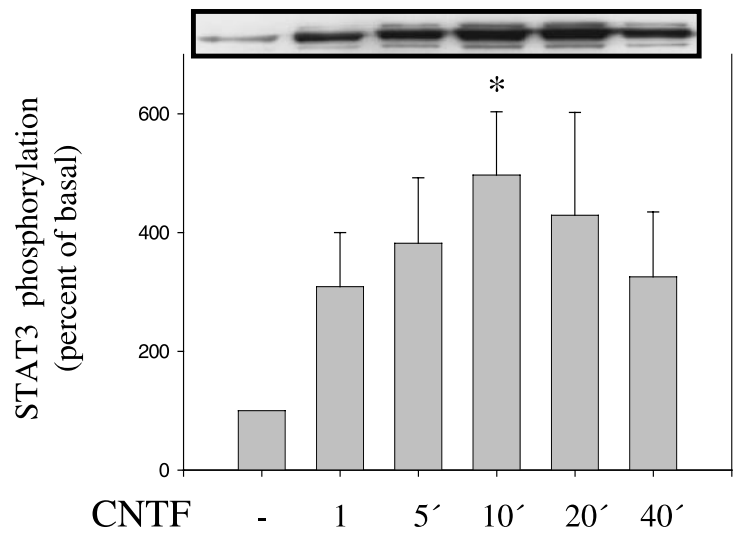

Figure 1 Ciliary neurotrophic factor (CNTF) induces STAT3 phosphorylation in a time- and dose-dependent manner. Following starvation for $48 \mathrm{~h}$ in serum-free medium cells were incubated with different concentrations of CNTF for $10 \mathrm{~min}(\mathrm{~A})$ and for various time periods at a CNTF concentration of $10 \mathrm{nM}$ (B), respectively. Protein lysates were prepared and immunoblotted using a phosphospecific STAT3 (Tyr 705) antibody. A representative immunoblot showing the band of interest $(80 \mathrm{kDa})$ and a bar graph analysis including the S.E.M. of three to six independent experiments are shown. ${ }^{*} P<0.05$ comparing non-stimulated to CNTF-treated cells.
Ag490 $(100 \mu \mathrm{M})$ led to an almost complete abrogation of CNTF's stimulatory effect on Akt phosphorylation (Fig. 3C). Similarly, inhibition of PI3-kinase (LY294002, $10 \mu \mathrm{M})$ potently blocked CNTF's effect on Akt activation (Fig. 3C). However, inhibition of mammalian target of rapamycin (mTor) (rapamycin, $10 \mathrm{nM}$ ) did not significantly alter the CNTF effect (Fig. 3C). By contrast and similar to PI3-kinase, inhibition of PKC (GF109203X, $5 \mu \mathrm{M})$ decreased the CNTF-induced Akt activation by about $70 \%$ (Fig. 3D).

CNTF induces p70 S6 kinase phosphorylation in a PI3-kinase-, mTor-, and PKC-dependent manner

CNTF treatment of brown adipocytes resulted in a dose- and time-dependent effect on p70 S6 kinase phosphorylation, with a fivefold stimulation after treatment with $10 \mathrm{nM}$ CNTF for $10 \mathrm{~min}$ (Fig. 4A and B). As seen previously, JAK2 inhibition with $\operatorname{Ag} 490 \quad(100 \mu \mathrm{M})$ decreased the CNTF-mediated p70 S6 kinase phosphorylation by approximately 90\% (Fig. 4C). PI3-kinase inhibition (LY294002, $10 \mu \mathrm{M}$ ) also diminished the CNTFmediated p70 S6 kinase phosphorylation by 80\% (Fig. 4C). In contrast to Akt activation, mTor inhibition by rapamycin $(10 \mathrm{nM})$ similarly reduced the CNTF-induced p70 S6 kinase phosphorylation by about 50\% (Fig. 4C). Again, PKC inhibition (GF109203X, $5 \mu \mathrm{M}$ ) reduced the CNTF-induced p70 S6 kinase phosphorylation by approximately 90\% (Fig. 4D).

\section{CNTF enhances $\beta 3$-adrenergic induction of UCP-1 expression}

To test effects of CNTF on a functional level which is specific to brown adipocytes and important to the regulation of energy homeostasis, we assessed UCP-1 expression in differentiated cells. After $2 \mathrm{~h}$ of treatment with $100 \mathrm{nM}$ of the selective $\beta 3$-adrenoceptor agonist CL316243 there was an approximately 2-fold increase in UCP-1 mRNA expression as compared with basal levels (Fig. 5). This increase was potently and significantly enhanced by pretreatment of adipocytes with CNTF $(10 \mathrm{nM})$ for $2 \mathrm{~h}$. The same effect was seen on the protein level (see inset, Fig. 5). CNTF treatment alone did not have a positive effect on basal UCP-1 mRNA expression but rather decreased it (Fig. 5). Furthermore, we did not find an effect of CNTF treatment on adipocyte differentiation or insulin-induced glucose uptake (data not shown).

\section{Discussion}

This study demonstrates direct and potent peripheral effects of CNTF on adipose tissue signalling and metabolism. Recent studies have elucidated an anorexigenic role 
A

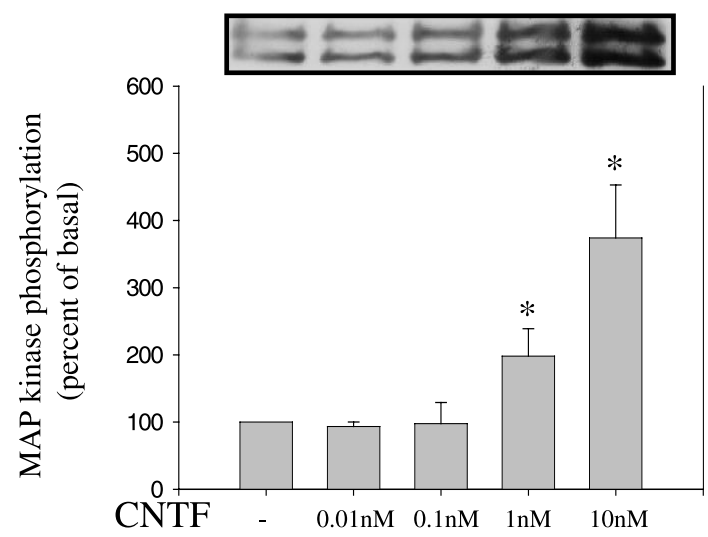

$\mathbf{C}$

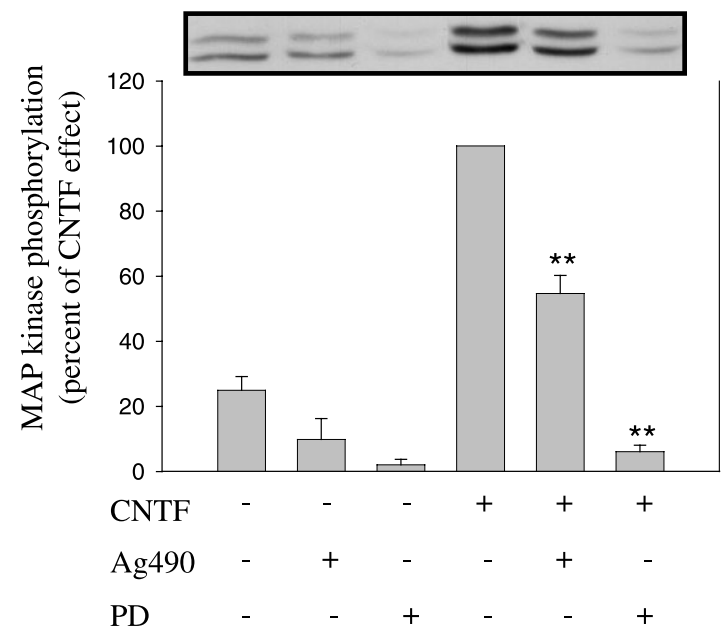

B

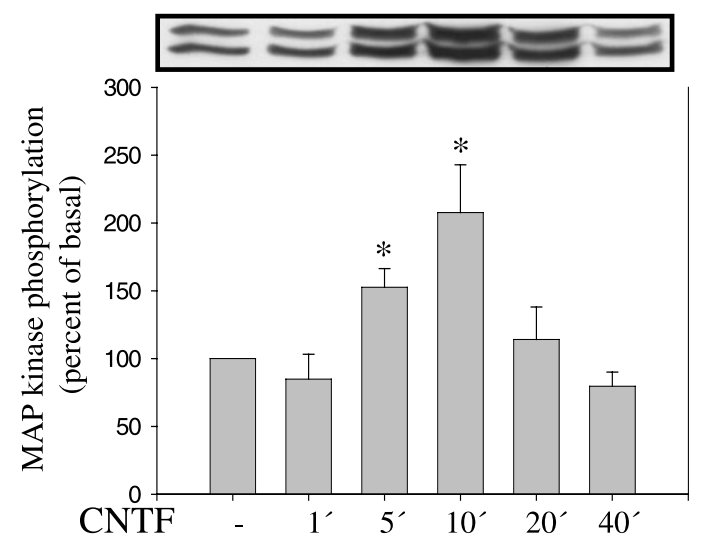

D

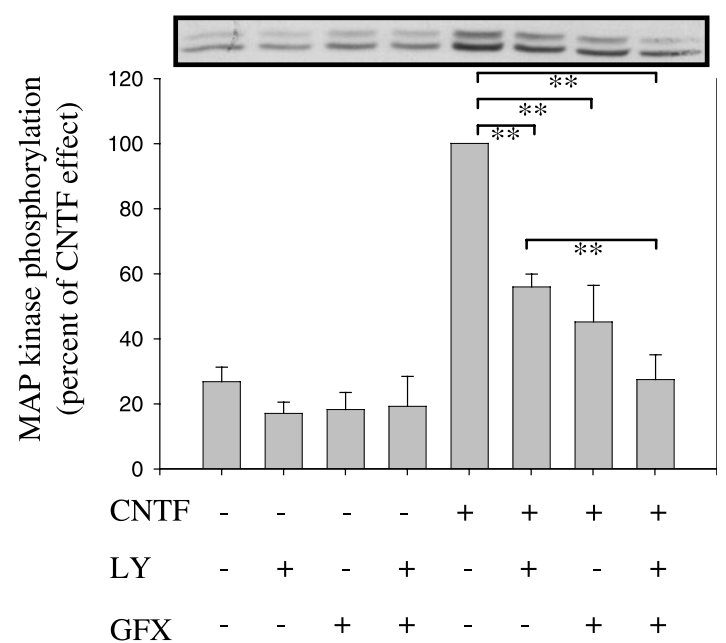

Figure 2 CNTF-induced activation of MAP kinase is JAK2-, PKC-, and PI3-kinase-dependent. After serum-starvation for $48 \mathrm{~h}$ cells were incubated with different concentrations of CNTF for $10 \mathrm{~min}(\mathrm{~A})$ and for various time periods at a CNTF concentration of $10 \mathrm{nM}(\mathrm{B})$, respectively. Furthermore, cells were pretreated with the pharmacological inhibitors as indicated prior to CNTF application (C, D). Pretreatment with the JAK2-inhibitor Ag490 (100 M), the MEK kinase inhibitor PD98059 ('PD', 10 M), the PKC inhibitor GF109203X ('GFX', $5 \mu \mathrm{M}$ ) and the PI3-kinase inhibitor LY294002 ('LY', $10 \mu \mathrm{M}$ ) was for 60 min respectively. MAP kinase phosphorylation was assessed by immunoblotting cell lysates with a phosphospecific antibody to p44/p42 MAP kinase. A bar graph analysis together with a representative immunoblot showing the bands of interest (42 and $44 \mathrm{kDa}$, respectively) are shown including the S.E.M. of three to six independent experiments. ${ }^{*} P<0 \cdot 05$, ${ }^{*} P<0 \cdot 01$ comparing non-stimulated to CNTF-treated cells (A, B) and CNTF-treated to inhibitor-treated cells (C), respectively, unless indicated otherwise (D).

for CNTF in the central nervous system regulation of energy homeostasis (Gloaguen et al. 1997, Lambert et al. 2001). To our knowledge however, this is the first report to demonstrate that CNTF selectively and potently activates main growth factor and metabolic signalling pathways in a peripheral tissue which is of primary importance to the regulation of body weight and insulin resistance. Moreover, CNTF appears to enhance $\beta 3$-adrenergic induction of UCP-1, the brown adipose tissue-specific protein which generates heat instead of ATP by uncoupling oxidative phosphorylation in the mitochondria (Klaus 1997, Nicholls et al. 1984). This suggests a role for 


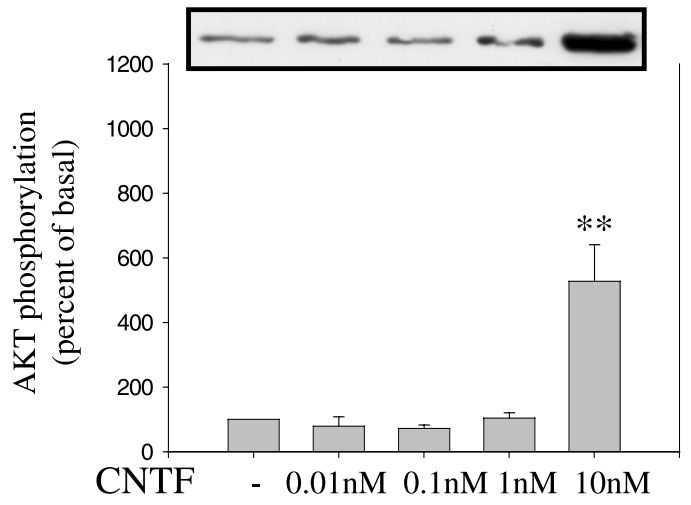

$\mathbf{C}$

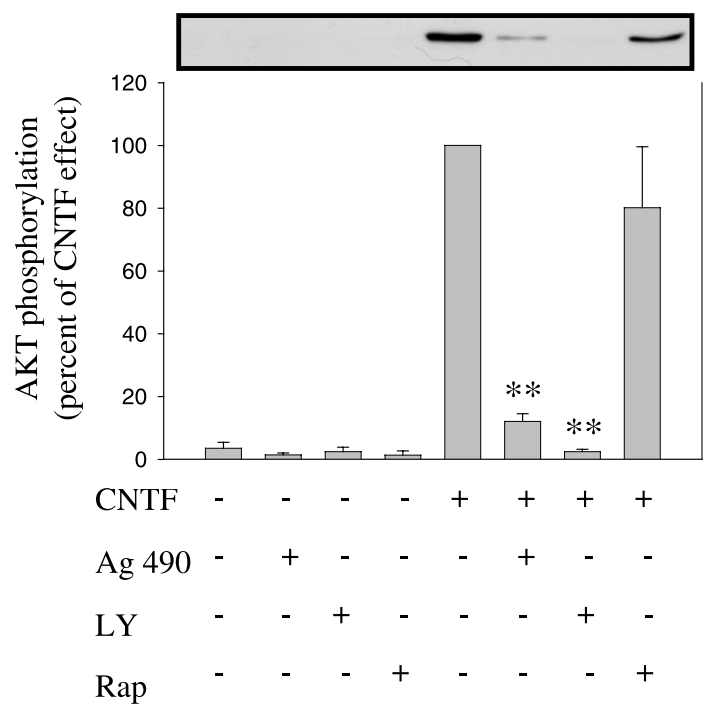

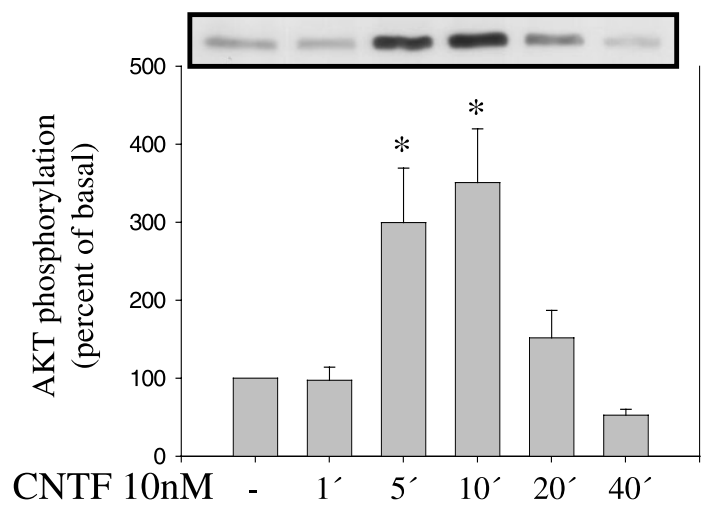

D

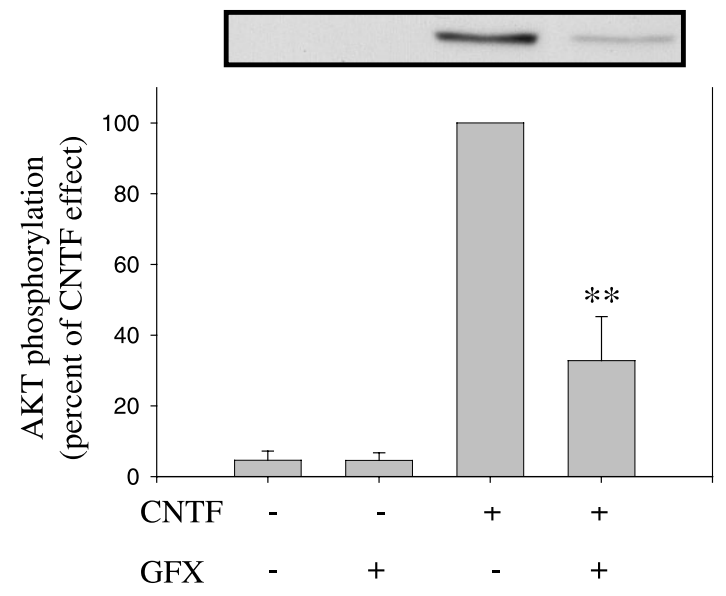

Figure 3 CNTF-mediated Akt phosphorylation is dependent on JAK2, PKC and PI3-kinase, but not mTor. Cells were serum-starved for $48 \mathrm{~h}$ and exposed to different concentrations of CNTF for $10 \mathrm{~min}(\mathrm{~A})$ and for various time periods at a CNTF concentration of $10 \mathrm{nM}(\mathrm{B})$, respectively. Furthermore, cells were pretreated with the indicated inhibitors prior to CNTF application (C, D). Pretreatment with Ag490 (100 $\mu$ M), GF109203X ('GFX', $5 \mu$ M) and LY294002 ('LY', $10 \mu M)$ was for 60 min, with rapamycin ('Rap', $10 \mathrm{nM}$ ) for $15 \mathrm{~min}$. Akt phosphorylation was assessed by immunoblotting cell lysates with a phosphospecific antibody to Akt (Ser 473). A representative immunoblot showing the band of interest (60 kDa) and a bar graph analysis of three to seven independent experiments including the S.E.M. are depicted. ${ }^{*} P<0 \cdot 05,{ }^{*} P<0 \cdot 01$ comparing non-stimulated to CNTF-treated cells (A, B) and CNTF-treated to inhibitor-treated cells (C, D) respectively.

CNTF in the peripheral regulation of energy homeostasis. Both white and brown adipose tissue have become targets of intense research to investigate physiological and molecular mechanisms leading to insulin resistance, as adipocyte metabolism is closely linked to maintaining energy homeostasis (Kahn et al. 2000, Lowell et al. 1997, Spiegelman et al. 2001). We have previously shown that immortalized brown adipocytes provide an excellent model system to investigate mechanisms leading to insulin resistance and dysregulation of energy balance (Fasshauer et al. 2000, Fasshauer et al. 2001a, Klein et al. 1999, Klein et al. 2000, Klein et al. 2002).

The present findings identify both important growth factor and metabolic signalling intermediates such as MAP 
A

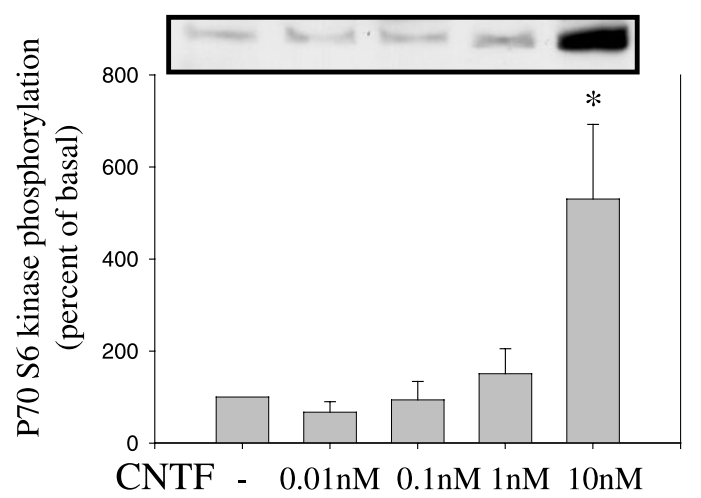

C

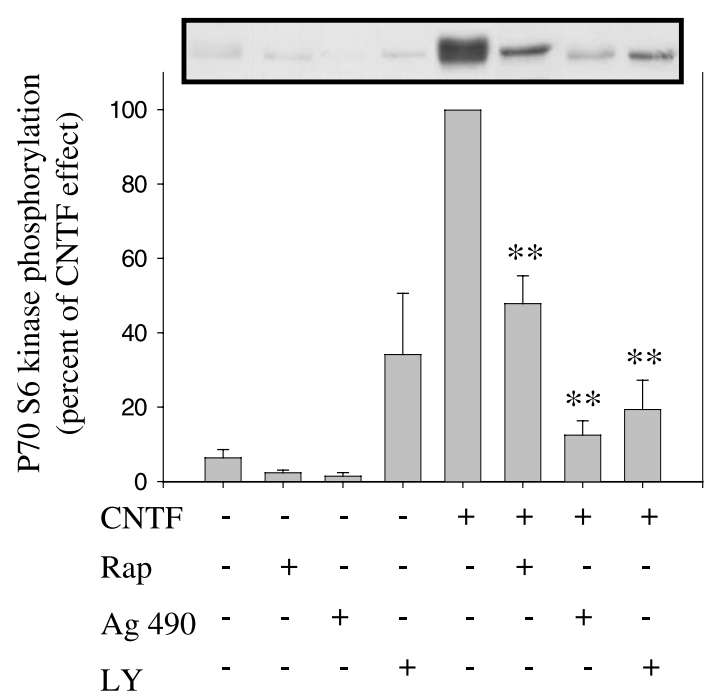

B

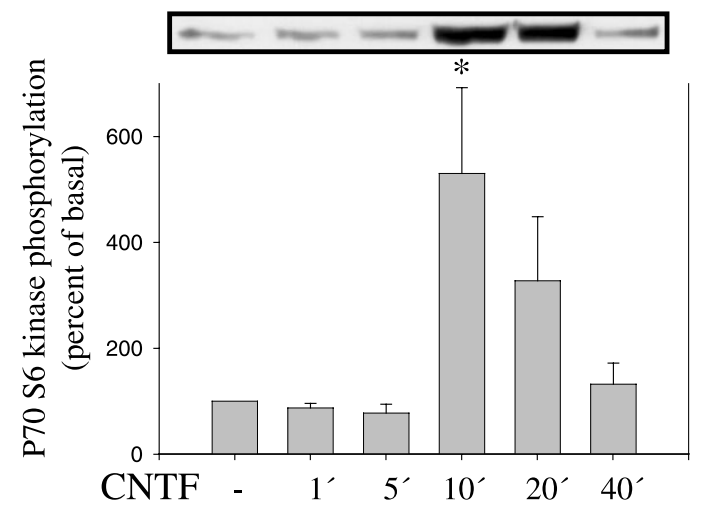

D

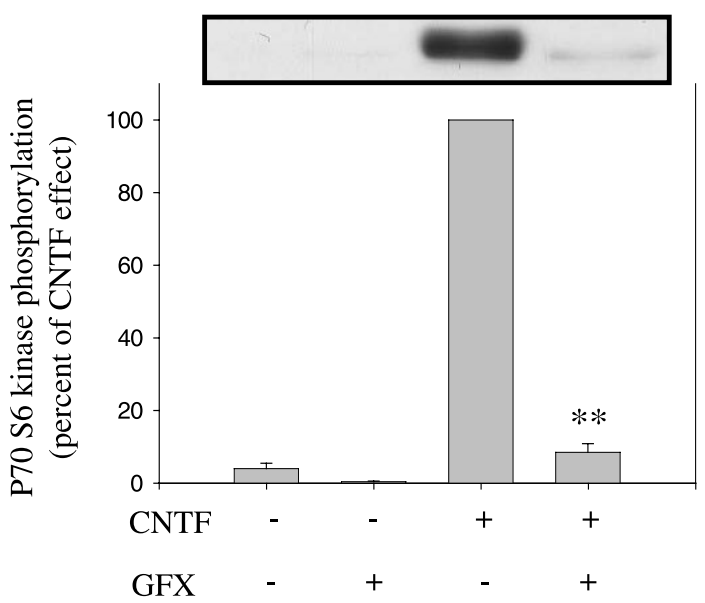

Figure 4 CNTF induces p70 S6 kinase via PI3-kinase-, PKC-, and mTor-dependent pathways. After serum-starvation for $48 \mathrm{~h}$ cells were incubated with different concentrations of CNTF for $10 \mathrm{~min}(\mathrm{~A})$ and for various time periods at a CNTF concentration of $10 \mathrm{nM}(\mathrm{B})$, respectively. Furthermore, cells were pretreated with the pharmacological inhibitors as indicated prior to CNTF application (C, D). Pretreatment with Ag490 (100 $\mu \mathrm{M})$, GF109203X ('GFX', $5 \mu \mathrm{M})$ and LY294002 ('LY', $10 \mu \mathrm{M})$ was for 60 min, with rapamycin ('Rap', $10 \mathrm{nM}$ ) for $15 \mathrm{~min}$. P70 S6 kinase phosphorylation was assessed by immunoblotting cell lysates with a phosphospecific antibody to p70 S6 kinase(Thr421/Ser 424). A representative immunoblot showing the band of interest $(70 \mathrm{kDa})$ and a bar graph analysis of three to eight independent experiments including the S.E.M. are depicted. ${ }^{*} P<0 \cdot 05$,

${ }^{*} P<0 \cdot 01$ comparing non-stimulated to CNTF-treated cells $(\mathrm{A}, \mathrm{B})$ and CNTF-treated to inhibitor-treated cells $(\mathrm{C}, \mathrm{D})$ respectively.

kinase, PI3-kinase, Akt, and p70 S6 kinase as molecular targets for direct CNTF activation in brown adipocytes, and delineate respective signalling cascades. CNTF causes a robust increase in STAT3 phosphorylation in brown adipocytes which is consistent with findings in a variety of neuronal cell-populations (Rajan 1996, Wishingrad et al. 1997). Furthermore, while there is no effect on p38 MAP kinase activation, CNTF selectively activates p42/44 MAP kinase. This activation appears partially JAK2dependent which is in accordance with a report from neuroblastoma cells (Lelievre et al. 2001).

Interestingly, both PI3-kinase and PKC inhibition additively inhibit CNTF-induced MAP kinase activation in brown adipocytes, thereby placing PI3-kinase upstream of 


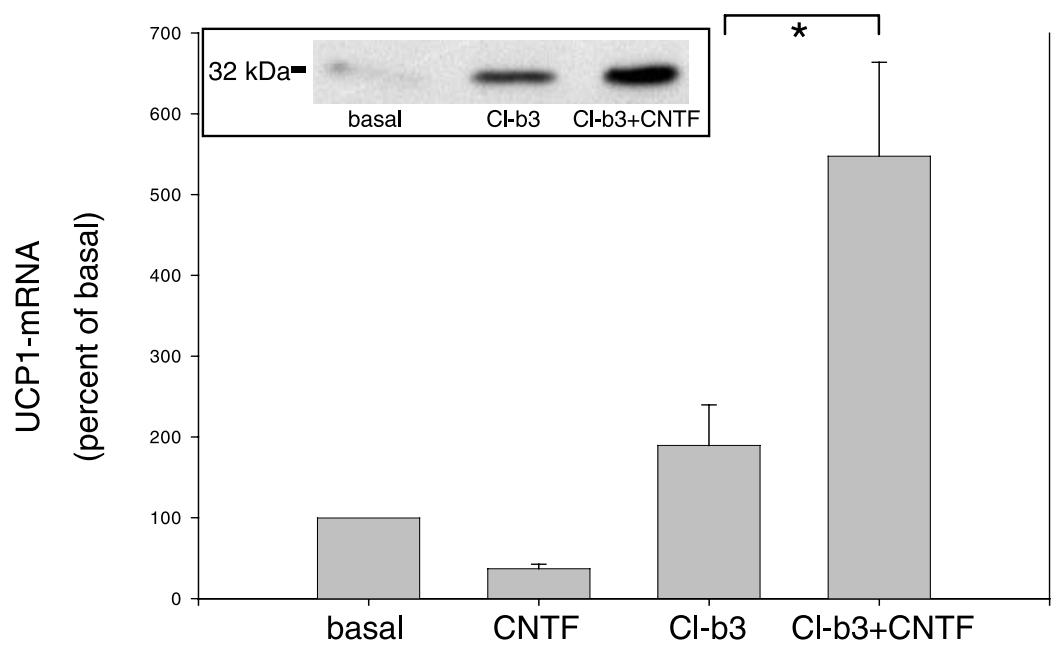

Figure 5 CNTF enhances $\beta 3$-adrenergic induction of UCP-1 expression. Differentiated brown adipocytes were stimulated for $2 \mathrm{~h}$ with the selective $\beta 3$-adrenoceptor agonist CL316243 (Cl-b3, $100 \mathrm{nM}$ ) either alone or after pretreatment of cells for $2 \mathrm{~h}$ with CNTF $(10 \mathrm{nM})$. CNTF treatment alone was for $4 \mathrm{~h}$. Total RNA was isolated and subjected to quantitative real-time RT-PCR as described in Materials and Methods. A bar graph analysis of three independent experiments including the S.E.M. is depicted. * denotes $P<0.05$ comparing treatment with the $\beta 3$-adrenoceptor agonist alone to combined treatment with the $\beta 3$-adrenoceptor agonist and CNTF. Inset: immunoblot showing the band of interest ( $32 \mathrm{kDa}$ ) using a UCP-1-specific antibody from cells treated as indicated (10 nM CNTF $2 \mathrm{~h}$ prior to Cl-b3 $100 \mathrm{nM}$ for $22 \mathrm{~h}$ ).

MAP kinase and attributing separate roles to PI3-kinase and $\mathrm{PKC}$ in the CNTF signalling cascades leading to MAP kinase activation. PI3-kinase and PKC dependence of MAP kinase activation has not been reported for CNTF signalling so far. Our results suggest further that PI3kinase and PKC-dependent pathways represent the main routes of CNTF-induced MAP kinase activation, as the combination of inhibitors for both signalling molecules completely suppresses the CNTF effect on MAP kinase.

CNTF also induces Akt and p70 S6 kinase phosphorylation in a dose- and time- dependent manner. The almost complete abrogation of CNTF effects by JAK2 inhibition with $\mathrm{Ag} 490$ in our experiments is in agreement with other studies implicating the JAK/Tyk family of kinases in IL6-family-cytokine signalling (Narazaki et al. 1994, Stahl 1994). Treatment with the selective PI3kinase inhibitor LY294002 significantly inhibits both Akt and p70 S6 kinase phosphorylation, while treatment with the selective mTor inhibitor rapamycin significantly inhibits p70 S6 kinase, but not Akt phosphorylation. This is concordant with a recent study by $\mathrm{Oh} e t$ al. on the relative positions of Akt and p70 S6 kinase in signalling cascades stimulated by IL6-family cytokines (Oh et al. 1998). Inhibition of PKC potently inhibits CNTF-induced Akt and p70 S6 kinase phosphorylation. Although not shown for CNTF signalling, this correlates with other reports showing a PKC dependence of Akt and p70 S6 kinase activation (Matsumoto et al. 2001, Romanelli et al. 1999).
While it appears plausible that the decrease in Akt phosphorylation resulting from PKC inhibition should extend downstream to p70 S6 kinase, it should be noted that there is evidence for a direct inhibition of p70 S6 kinase by the PKC inhibitor GF109203X (Alessi 1997).

Interestingly, activation of signalling pathways in brown adipocytes, on a functional level, corresponds with CNTFmediated enhancement of $\beta 3$-adrenergic induction of UCP-1. As UCP-1 is a mitochondrial uncoupler of oxidative phosphorylation and, therefore, contributes to a negative energy balance by producing heat instead of ATP, this suggests an important peripheral effect of CNTF on the control of energy homeostasis. However, the effect may be part of a complex signalling network, since CNTF treatment alone appears to decrease basal UCP-1 expression. In support of a selective role of CNTF on adipocyte metabolism we do not find alterations of insulininduced glucose uptake and adipocyte differentiation in CNTF-treated cells.

In summary, this study characterizes direct peripheral effects of the centrally acting anorexigenic neuropeptide CNTF on multiple growth factor and metabolic signalling pathways in brown adipose tissue. Furthermore, CNTF potently augments $\beta 3$-adrenergic stimulation of UCP-1 expression. This may indicate an important role of adipose tissue in mediating CNTF's negative effects on energy balance. 


\section{Acknowledgements}

This work was supported by grants from the Deutsche Forschungsgemeinschaft (K11131/2-1) and the Deutsche Diabetes Gesellschaft to Johannes Klein. We are indebted to Britta Meier for her excellent technical assistance. We gratefully acknowledge Dr. Kurt Steiner (Wyeth Ayerst Research, CN 8000, Princeton, NY, USA) for the kind gift of the $\beta 3$-adrenoceptor agonist CL316243.

\section{References}

Alessi DR 1997 The protein kinase C inhibitors Ro 318220 and GF 109203X are equally potent inhibitors of MAPKAP kinase- 1 beta (Rsk-2) and p70 s6 kinase. FEBS Letters 402 121-123.

ALS CNTF Treatment Study Group 1996 A double-blind placebo-controlled clinical trial of subcutaneous recombinant human ciliary neurotrophic factor (rHCNTF) in amyotrophic lateral sclerosis. Neurology 46 1244-1249.

Fasshauer M, Klein J, Ueki K, Kriauciunas KM, Benito M, White MF \& Kahn CR 2000 Essential role of insulin receptor substrate-2 in insulin stimulation of glut4 translocation and glucose uptake in brown adipocytes. Journal of Biological Chemistry 275 25494-25501.

Fasshauer M, Klein J, Kriauciunas KM, Ueki K, Benito M \& Kahn CR 2001a Essential role of insulin receptor substrate 1 in differentiation of brown adipocytes. Molecular and Cellular Biology 21 319-329.

Fasshauer M, Klein J, Neumann S, Eszlinger M \& Paschke R $2001 b$ Adiponectin gene expression is inhibited by beta-adrenergic stimulation via protein kinase a in 3T3-L1 adipocytes. FEBS Letters 507 142-146.

Fasshauer M, Klein J, Neumann S, Eszlinger M \& Paschke R 2001c Isoproterenol inhibits resistin gene expression through a $\mathrm{g}(\mathrm{s})$-proteincoupled pathway in 3T3-L1 adipocytes. FEBS Letters $\mathbf{5 0 0}$ 60-63.

Gloaguen I, Costa P, Demartis A, Lazzaro D, Di Marco A, Graziani R, Paonessa G, Chen F, Rosenblum CI, Van der Ploeg LHT, Cortese R, Ciliberto G \& Laufer R 1997 Ciliary neurotrophic factor corrects obesity and diabetes associated with leptin deficiency and resistance. PNAS 94 6456-6461.

Kahn BB \& Flier JS 2000 Obesity and insulin resistance. Journal of Clinical Investigation 106 473-481.

Klaus S 1997 Functional differentiation of white and brown adipocytes. Bioessays 19 215-223.

Klein J, Fasshauer M, Ito M, Lowell BB, Benito M \& Kahn CR 1999 Beta 3-adrenergic stimulation differentially inhibits insulin signaling and decreases insulin-induced glucose uptake in brown adipocytes. Journal of Biological Chemistry 274 34795-34802.

Klein J, Fasshauer M, Benito M \& Kahn CR 2000 Insulin and the beta3-adrenoceptor differentially regulate uncoupling protein-1 expression. Molecular Endocrinology 14 764-773.

Klein J, Fasshauer M, Klein HH, Benito M \& Kahn CR 2002 Novel adipocyte lines from brown fat: A model system for the study of differentiation, energy metabolism, and insulin action. BioEssays 24 382-388.

Lambert PD, Anderson KD, Sleeman MW, Wong V, Tan J, Hijarunguru A, Corcoran TL, Murray JD, Thabet KE,
Yancopoulos GD \& Wiegand SJ 2001 From the cover: Ciliary neurotrophic factor activates leptin-like pathways and reduces body fat, without cachexia or rebound weight gain, even in leptin-resistant obesity. PNAS 98 4652-4657.

Lelievre E, Plun-Favreau H, Chevalier S, Froger J, Guillet C, Elson GC, Gauchat JF \& Gascan H 2001 Signaling pathways recruited by the cardiotrophin-like cytokine/cytokine-like factor-1 composite cytokine: Specific requirement of the membrane-bound form of ciliary neurotrophic factor receptor alpha component. Journal of Biological Chemistry 276 22476-22484.

Lowell BB \& Flier JS 1997 Brown adipose tissue, beta 3-adrenergic receptors, and obesity. Annual Review of Medicine 48 307-316.

Matsumoto M, Ogawa W, Hino Y, Furukawa K, Ono Y, Takahashi M, Ohba M, Kuroki T \& Kasuga M 2001 Inhibition of insulininduced activation of Akt by a kinase-deficient mutant of the epsilon isozyme of protein kinase C. Journal of Biological Chemistry 276 14400-14406.

Narazaki M, Witthuhn B, Yoshida K, Silvennoinen O, Yasukawa K, Ihle J, Kishimoto T \& Taga T 1994 Activation of Jak2 kinase mediated by the interleukin 6 signal transducer gp130. PNAS 91 2285-2289.

Nicholls DG \& Locke RM 1984 Thermogenic mechanisms in brown fat. Physiological Review 64 1-64.

Oh H, Fujio Y, Kunisada K, Hirota H, Matsui H, Kishimoto T \& Yamauchi-Takihara K 1998 Activation of phosphatidylinositol 3-kinase through glycoprotein 130 induces protein kinase B and p70 S6 kinase phosphorylation in cardiac myocytes. Journal of Biological Chemistry 273 9703-9710.

Rajan P, Symes AJ \& Fink JS 1996 Stat proteins are activated by ciliary neurotrophic factor in cells of central nervous system origin. Journal of Neuroscience Research 43 403-411.

Romanelli A, Martin KA, Toker A \& Blenis J 1999 P70 S6 kinase is regulated by protein kinase Czeta and participates in a phosphoinositide 3-kinase-regulated signalling complex. Molecular and Cellular Biology 19 2921-2928.

Sleeman MW, Anderson KD, Lambert PD, Yancopoulos GD \& Wiegand SJ 2000 The ciliary neurotrophic factor and its receptor, CNTFR alpha. Pharmaceutica Acta Helvetiae 74 265-272.

Spiegelman BM \& Flier JS 2001 Obesity and the regulation of energy balance. Cell 104 531-543.

Stahl N, Boulton TG, Farruggella T, Ip NY, Davis S, Witthuhn BA, Quelle FW, Silvennoinen O, Barbieri G, Pellegrini S et al. 1994 Association and activation of Jak-Tyk kinases by CNTF-LIF-OSM-IL-6 beta receptor components. Science 263 92-95.

Wishingrad MA, Koshlukova S \& Halvorsen SW 1997 Ciliary neurotrophic factor stimulates the phosphorylation of two forms of STAT3 in chick ciliary ganglion neurons. Journal of Biological Chemistry 272 19752-19757.

Xu B, Dube MG, Kalra PS, Farmerie WG, Kaibara A, Moldawer LL, Martin D \& Kalra SP 1998 Anorectic effects of the cytokine, ciliary neurotropic factor, are mediated by hypothalamic neuropeptide Y: Comparison with leptin. Endocrinology 139 466-473.

Received 23 February 2002

Accepted 12 April 2002 